\title{
Estimated GFR and Mortality in Older Men: Are All eGFR Formulae Equal?
}

\author{
Muna T. Canales ${ }^{a}$ Terri Blackwell ${ }^{c}$ Areef Ishani $^{d}$ Brent C. Taylor ${ }^{\mathrm{e}}$ \\ Allyson Hart ${ }^{9}$ Elizabeth Barrett-Connor ${ }^{\text {h }}$ Cora Lewis $^{\mathrm{i}}$ Rebecca J. Beyth ${ }^{\text {b }}$ \\ Katie Stone $^{c}$ Kristine E. Ensrud $^{f}$ for the Outcomes of Sleep Disorders in Older \\ Men (MrOS Sleep) Study

\begin{abstract}
a Department of Medicine, Malcom-Randall VAMC, and b Department of Medicine, Malcom-Randall VAMC GRECC, University of Florida, Gainesville, Fl., 'Research Institute, California Pacific Medical Center, San Francisco, Calif., ${ }^{\mathrm{d}}$ Department of Medicine, VAMC, e Department of Medicine, Center for Chronic Disease Outcomes Research, VAMC, and ${ }^{f}$ Department of Medicine, University of Minnesota, and ${ }^{9}$ Hennepin County Medical Center, Minneapolis, Minn., ${ }^{h}$ Department of Family Medicine and Public Health, University of California, San Diego, Calif., 'Department of Medicine, University of Alabama, Birmingham, Ala., USA
\end{abstract}

\section{Key Words}

Chronic kidney disease - Geriatric nephrology · Glomerular filtration rate $\cdot$ Estimation of renal function - Mortality

\footnotetext{
Abstract

Background: Recently, the first estimated glomerular filtration rate (eGFR) formula specifically developed for community-dwelling older adults, the Berlin Initiative Study Equation 2 (BIS2), was reported. To date, however, no study has examined the performance of the BIS2 to predict death in older adults as compared to equations used clinically and in research. Methods: We prospectively followed 2,994 community-dwelling men (age $76.4 \pm 5.6$ ) enrolled in the MrOS Sleep Study. We calculated baseline eGFR from serum creatinine and cystatin-C using the BIS2, Chronic Kidney Disease Epidemiology (CKD-EPI $\left.{ }_{\mathrm{cr}, \mathrm{cysc}}\right), \mathrm{CKD}-\mathrm{EPI}_{\mathrm{cysc}}$ and CKD-EPI ${ }_{\mathrm{cr}}$ equations. Analyses included Cox-proportional hazards regression and net reclassification improvement (NRI) for the
}

outcomes of all-cause and cardiovascular death. Results: Follow-up time was $7.3 \pm 1.9$ years. By BIS2, 42 and $11 \%$ had eGFR $<60$ and $<45$, respectively, compared to CKD-EPI cr $(23$ and 6\%), CKD-EPI cysc $_{\text {(36 and 13\%) and CKD-EPI }}$ cr,ysc (28 and $8 \%)$. BIS2 eGFR $<45$ was associated with twofold higher rate of all-cause mortality when compared to eGFR $\geq 75$ after multivariate adjustment (HR 2.1,95\% Cl 1.5-2.8). Results were similar for CKD-EPI ${ }_{\mathrm{Cr}, \mathrm{cysc}}<45$ (HR 2.1,95\% Cl 1.6-2.7) and CKD$\mathrm{EPI}_{\text {cysc }}<45$ (HR 2.1, 95\% Cl 1.7-2.7) and weaker for CKD-EPI ${ }_{\text {cr }}$ $<45$ (HR 1.5, 95\% Cl 1.2-2.0). In NRI analyses, when compared to CKD-EPI ${ }_{\mathrm{Cr}, \text {,ysc }}$ both BIS2 and CKD-EPI ${ }_{\mathrm{cr}}$ equations more often misclassified participants with respect to mortality. We found similar results for cardiovascular death. Conclusion: The BIS2 did not outperform and the CKD-EPI ${ }_{\mathrm{Cr}}$ was inferior to the cystatin C-based CKD-EPI equations to predict death in this cohort of older men. Thus, the cystatin C-based CKD$\mathrm{EPI}$ equations are the formulae of choice to predict death in community-dwelling older men.

(c) 2016 S. Karger AG, Basel

\section{KARGER}

E-Mail karger@karger.com www.karger.com/ajn
(C) 2016 S. Karger AG Basel

$0250-8095 / 16 / 0435-0325 \$ 39.50 / 0$
Muna T Canales, MD, MS

Department of Medicine

University of Florida, Mail code 111G

1601 SW Archer Road, Gainesville, FL 32608 (USA)

E-Mail muna.canales@medicine.ufl.edu 


\section{Introduction}

Nearly 1 in 4 Americans over 60 years of age in the United States have chronic kidney disease (CKD) defined by an estimated glomerular filtration rate (eGFR) of $<60$ $\mathrm{ml} / \mathrm{min} / 1.73 \mathrm{~m}^{2}[1]$. Older adults with CKD suffer high rates of morbidity and mortality, in excess of what is attributable to their age and comorbid conditions [2-4]. Studies of mortality risk among older adults suggest that $\mathrm{CKD}$ is an independent risk factor for death, including death due to cardiovascular events [4]. However, the level of renal function at which this increased risk begins is unclear, likely due to challenges in measurement of renal function in aged populations [4]. Specifically, among healthy older adults, the insensitivity of serum creatinine (SCr)-based measures to predict death and the lack of eGFR formulae developed for this population may lead to inaccurate risk estimates [5].

To address the insensitivity of SCr-based measures of GFR, newer equations have incorporated serum cystatin$\mathrm{C}$ (SCysc) alone or in combination with SCr in an effort to overcome some of the limitations of SCr-based measures [5]. The Chronic Kidney Disease Epidemiology (CKD-EPI) collaboration equations were developed using a pooled sample of participants from various studies to represent a range of ages and renal function as measured using SCysc and/or SCr [5]. Findings from the CKD-EPI collaboration suggest that among older adults, renal function as estimated by SCysc- or SCr/SCysc-based equations are more sensitive predictors of mortality than when estimated using SCr-based formulae [6]. However, questions remain about the predictive validity of these equations in the aged population as the mean age of the CKD-EPI cohorts was in the range $47-51$ with only $13 \%$ of subjects over 65 years [5].

Recently, in an effort to develop and validate an estimating equation in an older cohort, investigators in the Berlin Initiative Study (BIS) developed 2 novel equations, the BIS1 (SCr-based) and BIS2 (SCr and SCyscbased) equations, using as their gold standard a direct measurement of GFR in a random community-based sample of 570 men and women over age 70 enrolled in a large health insurance program [7]. This study reported that the BIS2 equation yielded the best estimate of measured GFR (mGFR) when compared to Modification of Diet and Renal Disease, Cockroft-Gault, CKD-EPI $I_{c r}$ and the 2008 CKD-EPI equations based upon SCysc (with and without age, gender adjustment) or combination of SCysc and SCr $[7,8]$. However, no study to date has compared the ability of the BIS2 equation to predict risk of all-cause and cardiovascular mortality in communitydwelling older adults to that of other estimating equations [7]. Therefore, using data collected in prospective multicenter cohort study of community-dwelling older men aged 67 years and older, we compared the ability of the BIS2 equation and other standard estimating equations used clinically and in research to that of the CKD$\mathrm{EPI}_{\mathrm{cr}, \mathrm{cysc}}$ equation to predict all-cause and cardiovascular mortality.

\section{Subjects and Methods}

\section{Participants}

From 2000 through $2002,5,994$ men who were $\geq 65$ years were recruited for participation in the baseline examination of the Osteoporotic Fractures in Men (MrOS) Study, the parent cohort for the MrOS Sleep Study $[9,10]$. The enrollment criteria were designed to recruit a cohort that represented characteristics of community-dwelling older men residing in the United States $[9,10]$. Men were recruited from population-based listings in 6 regions (Birmingham, Alabama; Minneapolis, Minnesota; Palo Alto, California; the Monongahela Valley near Pittsburgh, Pennsylvania; Portland, Oregon; and San Diego, California) $[9,10]$. Men with a history of bilateral hip replacement, men who were unable to walk without the assistance of another person and men with a medical condition (in the judgment of the investigator) that would result in imminent death were excluded $[9,10]$.

Between December 2003 and March 2005, a total of 3,135 men from MrOS were recruited for participation in the MrOS Sleep Study as part of an ongoing prospective study of consequences of sleep disorders in this population. Of the 3,135 men enrolled in MrOS Sleep, 3,034 participants had serum from the MrOS Sleep visit available for measurement of renal function. Of these men, 40 men were missing data on vital status, leaving 2,994 men in the final analytical cohort.

All men provided written informed consent, and the study was approved by the Institutional Review Board at each site.

\section{Measurement of Renal Function Biomarkers}

Measurement of renal function biomarkers was performed on previously frozen $\left(-70^{\circ} \mathrm{C}\right)$ stored serum samples from the $\mathrm{MrOS}$ Sleep visit; $\mathrm{SCr}$ and SCysc were measured from the same sample and freeze-thaw cycle between July 31, 2013 and August 23, 2013. SCr was analyzed using the Roche Modular P chemistry analyzer (Enzymatic/Roche Diagnostics Corp., Indianapolis, Ind., USA). This assay is calibrated to be isotope dilution mass spectrometry traceable. SCysc was measured using the Roche Modular P chemistry analyzer (Turbidimetric/Gentian AS, Moss, Norway). This assay is calibrated to the international cystatin C reference material ERM-DA471/ IFCC. Both assays were performed at the Fairview University Medical Center Clinical Laboratory, Minneapolis, Minn., USA. The interassay coefficient of variation (CV) for SCr is $3.7 \%$ at $0.82 \mathrm{mg} / \mathrm{dl}$ and $2.3 \%$ at $3.62 \mathrm{mg} / \mathrm{dl}$. For SCysc the inter-assay CV is $4.1 \%$ at $0.94 \mathrm{mg} / \mathrm{l}$ and $2.8 \%$ at $3.29 \mathrm{mg} / \mathrm{l}$. We estimated GFR using the CKD-EPI $\mathrm{cr}_{\mathrm{cr}}$,

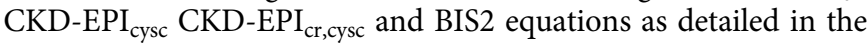
online supplementary appendix table 1 (for all online suppl. material, see www.karger.com/doi/10.1159/000445757) $[5,7,11]$. 
Table 1. Prevalence of eGFR category using 4 different estimating equations

\begin{tabular}{|c|c|c|c|c|}
\hline \multirow{2}{*}{$\begin{array}{l}\text { eGFR category, } \mathrm{ml} / \\
\mathrm{min} / 1.73 \mathrm{~m}^{2}\end{array}$} & \multicolumn{4}{|c|}{ GFR estimating equation, $\mathrm{n}(\%)$} \\
\hline & CKD-EPI ${ }_{\text {cr,cysc }}$ & BIS2 & $\mathrm{CKD}-\mathrm{EPI}_{\mathrm{cr}}$ & CKD-EPI $I_{\text {cysc }}$ \\
\hline$\geq 75$ & $1,205(40.2)$ & $517(17.3)$ & $1,320(44.1)$ & $1,078(36.0)$ \\
\hline $60-74$ & $939(31.4)$ & $1,217(40.7)$ & $978(32.7)$ & $826(27.6)$ \\
\hline $45-59$ & $602(20.1)$ & $943(31.5)$ & $522(17.4)$ & $691(23.1)$ \\
\hline$<45$ & $248(8.3)$ & $317(10.6)$ & $174(5.8)$ & $399(13.3)$ \\
\hline
\end{tabular}

\section{Mortality Outcome}

Participants were contacted every 4 months to ascertain vital status. During a follow-up of $7.3 \pm 1.9$ years, over $99 \%$ of these contacts were completed. Deaths were confirmed with death certificates and cause of death was adjudicated due to cardiovascular disease (International Classification of Diseases-9 codes 396.9$442,966.71,785.51)$ by central physician review of death certificates and medical records.

\section{Other Measurements}

Candidate variables included in our analysis were collected primarily from the baseline MrOS Sleep visit during 2003-2005. Race/ethnicity and education were determined from the baseline MrOS visit. Variables used in this study included age and race/ ethnicity, where race/ethnicity was defined as white, black or other (defined as Asian, Hispanic, Pacific Islander, American Indian or more than one race); body mass index $\left(\mathrm{BMI}, \mathrm{kg} / \mathrm{m}^{2}\right)$ as a measure of obesity as well as measures of lean body mass (total and appendicular lean muscle mass as determined by dual energy Xray absorptiometry, Hologic 4500 scanners, Waltham, Mass., USA); tobacco and alcohol use; self-reported health status based upon SF-12 questionnaire [12]; and medical history including hypertension (HTN: self-report or any anti-hypertensive medication use), diabetes mellitus (DM: self-report or any hypoglycemic medication use), self-reported peripheral vascular disease, self-reported coronary heart disease (CHD: myocardial infarction, angina, bypass or angioplasty), self-reported history of congestive heart failure $(\mathrm{CHF})$ or self-reported history of cerebrovascular disease (history of stroke).

\section{Statistical Analysis}

Our primary analysis compared the performance of the BIS2 equation with the CKD-EPI $\mathrm{cr}_{\text {, cysc }}$ equation to predict mortality. Thus, we examined baseline characteristics and potential confounders of the association between renal function and mortality across eGFR categories $\left(\geq 75,60-74,45-59\right.$ and $<45 \mathrm{ml} / \mathrm{min} / 1.73 \mathrm{~m}^{2}$; henceforth, eGFR units are removed for ease of reading) defined by

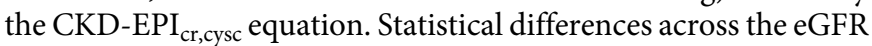
category were calculated using chi-square and analysis of variance tests for categorical and continuous variables, respectively. We also compared the prevalence of participants in each eGFR category using each of the 4 estimating equations with a chi-square test. Nonparametric kernel estimation densities of eGFR were plotted for each of the 4 estimating equations.

We determined all-cause and cardiovascular mortality rate (deaths/1,000 person-years) during an average of 7.3 years of fol- low-up by category of baseline eGFR for each estimating equation. Cox proportional hazards regression was used to determine hazard ratios for death. Based upon factors determined a priori with clinical relevance to be potential confounders of the association between renal function and mortality (age, race/ethnicity, BMI, DM, HTN) and other factors that were associated with both predictor and outcomes, we present the following models: (1) an unadjusted model (site only); (2) a base model, further adjusted for age, race/ethnicity and BMI; and (3) a final model, further adjusted for HTN, DM, CHF, CHD, and stroke.

Finally, in order to determine how all-cause and cardiovascular mortality for each eGFR category changed depending upon the estimating equation used, we calculated a metric comparable to the net reclassification improvement (NRI) for each formula compared to the CKD-EPI ${ }_{\mathrm{cr}, \mathrm{cysc}}$ (online suppl. appendix table 2 for NRI formulae) [13]. A positive NRI, which is the total for the estimating equation evaluated means that the equation more often appropriately reclassifies to a higher eGFR (better function) than those participants who lived and/or reclassifies to a lower eGFR (worsefunction) those who died when compared to the referent equation $\left(\mathrm{CKD}-\mathrm{EPI}_{\mathrm{cr}, \mathrm{cysc}}\right)$. We also present a category-free NRI, which defines reclassification as 1 unit change in eGFR [14]. The categoryfree NRI allows us to provide estimates that may be more comparable across studies including the eGFR categories we have chosen [14].

In secondary analyses, we repeated all of the above analyses expressing eGFR in quartiles. All significance levels reported were two sided and all analyses were conducted using SAS 9.4 (SAS Institute Inc., Cary, N.C., USA).

\section{Results}

\section{Baseline eGFR and Characteristics}

Fewer (17\%) participants were categorized as eGFR $\geq 75$ by BIS2 eGFR in contrast to 44,36 , and $40 \%$ for the CKD-EPI ${ }_{c r}, C K D-E P I_{c y s c}$ and CKD-EPI ${ }_{c r, c y s c}$ respectively ( $\mathrm{p}<0.001$ for all pairwise comparisons; table 1$)$. The BIS2 equation identified a larger proportion of participants as having eGFR $<60(42 \%)$ compared to 23,36 , and $28 \%$ with eGFR $<60$ for the CKD-EPI $I_{c r}, C K D-E P I_{c y s c}$ and CKD-EPI $I_{\text {cr,cysc }}$ respectively. The overrepresentation of eGFR $<60$ by BIS 2 was largely due to a higher prevalence 
Table 2. Clinical characteristics of MrOS Sleep participants by CKD-EPI $I_{c r, c y s c}$ eGFR category

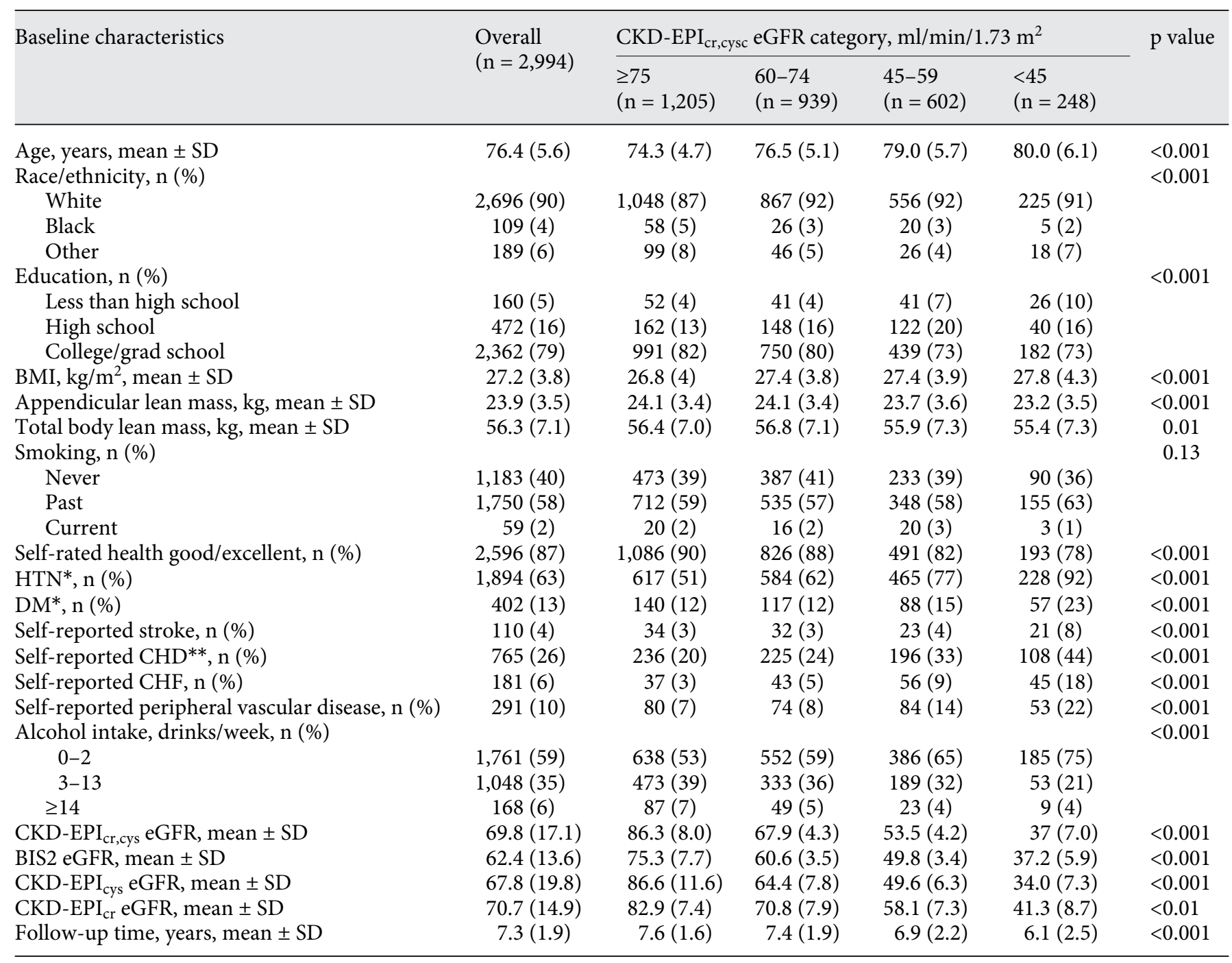

* As determined by self-report or any disease-specific medication use.

** Includes myocardial infarction, coronary artery bypass grafting, and angioplasty.

of eGFR $45-59$ (32\% for BIS2 vs. 17,23 , and $20 \%$ for the CKD-EPI $I_{c r}, C K D-E P I_{c y s c}$, and CKD-EPI $I_{c r, c y s c}$, respectively). Online suppl. appendix figure 1 depicts the distribution of eGFR by each equation graphically.

Men with lower CKD-EPI $I_{\text {cr,cysc }}$ eGFR or worse renal function were on average older, had higher BMI and lower total and appendicular lean body mass (table 2; see similar tables by BIS2, CKD-EPI ${ }_{c y s c}$ and CKD-EPI $\mathrm{cr}_{\mathrm{cr}}$ in online suppl. appendix tables 3-5). Also, men with lower CKD$\mathrm{EPI}_{\mathrm{cr}, \mathrm{cysc}}$ eGFR reported worse health status and had more HTN, DM, CHD, CHF, peripheral vascular disease and stroke.

\section{eGFR and Mortality}

During the average follow-up of $7.3 \pm 1.9$ years, 751 (25\%) men died. Overall, in unadjusted analyses, lower category of eGFR (worse renal function) using either CKD-EPI equation was associated with a graded increase in mortality (table 3 ). Participants with CKD-EPI cr,cysc $<45,45-59$, and 60-74 were 4.4, 2.2, and 1.5 times more likely to die, respectively, than those with CKD-EPI cr,cysc $\geq 75$ (p-trend $<0.001$ ). For those with eGFR $<45$ and $45-$ 59 this association persisted despite multivariate adjust-

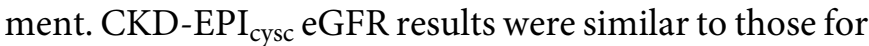
CKD-EPI ${ }_{\text {cr,cysc }}$ (table 3). In unadjusted analyses, BIS2 
Table 3. Association of eGFR and all-cause mortality

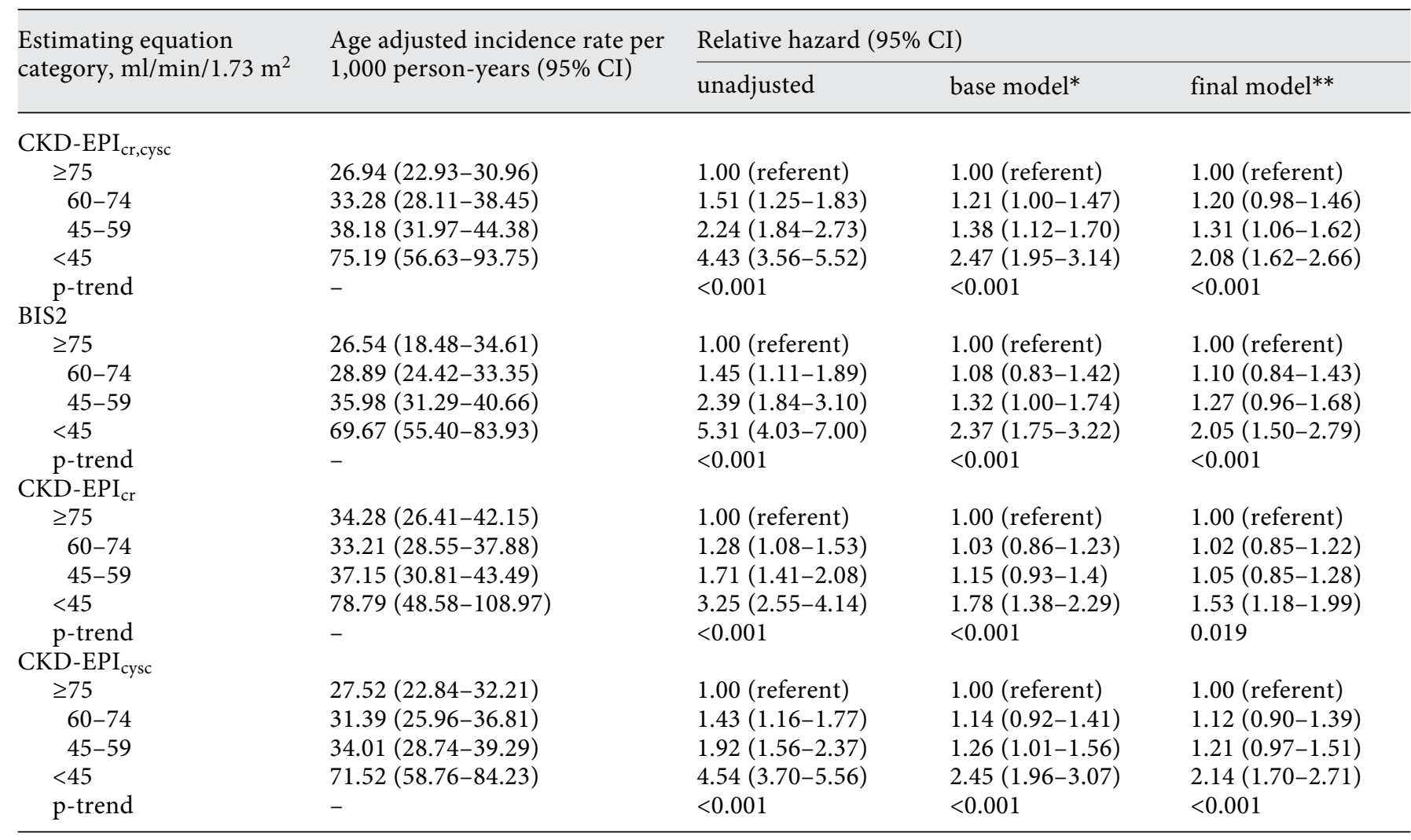

* Adjusted for age, race/ethnicity, and BMI.

** Adjusted for age, race/ethnicity, BMI, HTN, DM, CHF, CHD, and stroke.

eGFR $<45,45-59$ and 60-75 conferred a 5.3, 2.4 and 1.5fold greater risk of death when compared to eGFR $\geq 75$ (p-trend $<0.001$ ). After multivariate adjustment, this association was attenuated such that only eGFR $<45$ was associated with a statistically significant increased risk of death, though point estimates were similar to CKD-EPI equations. Finally, the hazard ratios for death by CKD$\mathrm{EPI}_{\mathrm{cr}}$ eGFR category and mortality followed a similar trend to the BIS2 and CKD-EPI analyses, though the point estimates were less robust (HR for death for CKDEPI $_{\text {cr }}$ eGFR $<45$ vs. $\geq 751.5$, p-trend $\left.=0.01\right)$. When cardiovascular mortality was the outcome, results were similar to those for all-cause mortality except that the point estimates for CKD-EPI ${ }_{\mathrm{cr}}$ eGFR as a predictor of cardiovascular death were comparable to the other 3 equations (online suppl. appendix table 6). In sensitivity analyses, when eGFR was categorized in quartiles instead of categories, we found overall similar results for the association between eGFR and mortality (online suppl. appendix tables 7 and 8).

eGFR and Death in Older Men
Finally, in order to determine the ability of the BIS2 formula to appropriately re-classify participants for risk of all-cause and cardiovascular death when compared to the

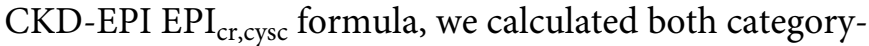
based and category-free NRI (table 4). For each CKD$\mathrm{EPI}_{\mathrm{cr}, \mathrm{cysc}}$ category, the BIS2 either made no change in category or reclassified participants down to a lower eGFR regardless of event status, except for one participant. For those who died (experienced an event), the recategorization to a lower eGFR by BIS2 reflected an appropriate and statistically significant reclassification. However, for those who did not die (no event), the recategorization to a lower eGFR by BIS2 (except for one participant) reflected an inappropriate and statistically significant reclassification. Because overall BIS2 reclassified more participants to lower eGFR inappropriately for their vital status, the net combination of the NRI for events and non-events was nega-

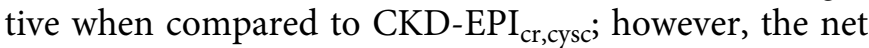
NRI was not statistically different from zero. A negative overall NRI value means that the BIS2 compared to CKD- 
Table 4. Net reclassification improvement for GFR estimating equations when compared to CKD-EPI $I_{c r, c y s c}$ equation

\begin{tabular}{|c|c|c|c|c|}
\hline $\begin{array}{l}\text { Estimating equation } \\
\text { by outcome }\end{array}$ & $\begin{array}{l}\text { Category-based* } \\
\text { NRI }(95 \% \text { CI })\end{array}$ & $\mathrm{p}$ value & $\begin{array}{l}\text { Category-free** } \\
\text { NRI }(95 \% \mathrm{CI})\end{array}$ & $\mathrm{p}$ value \\
\hline \multicolumn{5}{|l|}{ All-cause mortality } \\
\hline \multicolumn{5}{|l|}{ BIS2 } \\
\hline Events & $0.37(0.34$ to 0.40$)$ & $<0.0001$ & $0.81(0.77$ to 0.84$)$ & $<0.001$ \\
\hline Non-events & $-0.40(-0.42$ to -0.38$)$ & $<0.0001$ & $-0.94(-0.95$ to -0.92$)$ & $<0.001$ \\
\hline Total NRI & $-0.03(-0.07$ to 0.01$)$ & 0.312 & $-0.13(-0.16$ to -0.09$)$ & 0.002 \\
\hline \multicolumn{5}{|l|}{ CKD-EPI ${ }_{\mathrm{cr}}$} \\
\hline Events & $-0.24(-0.28$ to -0.20$)$ & $<0.0001$ & $-0.38(-0.44$ to -0.32$)$ & $<0.0001$ \\
\hline Non-events & $0.07(0.05$ to 0.09$)$ & $<0.0001$ & $0.04(0.004$ to 0.08$)$ & 0.03 \\
\hline Total NRI & $-0.17(-0.22$ to -0.13$)$ & $<0.0001$ & $-0.34(-0.41$ to -0.27$)$ & $<0.0001$ \\
\hline \multicolumn{5}{|l|}{ CKD-EPI $I_{\text {cysc }}$} \\
\hline Events & $0.23(0.20$ to 0.27$)$ & $<0.0001$ & $0.53(0.47$ to 0.59$)$ & $<0.001$ \\
\hline Non-events & $-0.15(-0.17$ to -0.13$)$ & $<0.0001$ & $-0.24(-0.28$ to -0.20$)$ & $<0.001$ \\
\hline Total NRI & 0.08 (0.04 to 0.12$)$ & 0.0002 & $0.29(0.22$ to 0.36$)$ & $<0.001$ \\
\hline \multicolumn{5}{|c|}{ Cardiovascular mortality } \\
\hline \multicolumn{5}{|c|}{ BIS2 } \\
\hline Events & $0.33(0.28$ to 0.39$)$ & $<0.0001$ & $0.76(0.69$ to 0.83$)$ & $<0.001$ \\
\hline Non-events & $-0.39(-0.41$ to -0.38$)$ & $<0.0001$ & $-0.91(-0.93$ to -0.90$)$ & $<0.001$ \\
\hline Total NRI & $-0.06(-0.12$ to 0.0004$)$ & 0.114 & $-0.16(-0.22$ to -0.09$)$ & 0.01 \\
\hline \multicolumn{5}{|l|}{ CKD-EPI ${ }_{\mathrm{cr}}$} \\
\hline Events & $-0.23(-0.31$ to -0.16$)$ & $<0.0001$ & $-0.40(-0.50$ to -0.29$)$ & $<0.0001$ \\
\hline Non-events & $0.10(0.08$ to 0.12$)$ & $<0.0001$ & $0.10(0.07$ to 0.14$)$ & $<0.0001$ \\
\hline Total NRI & $-0.13(-0.21$ to -0.06$)$ & 0.0012 & $-0.29(-0.40$ to -0.18$)$ & $<0.0001$ \\
\hline \multicolumn{5}{|l|}{ CKD-EPI $I_{\text {cysc }}$} \\
\hline Events & $0.24(0.18$ to 0.29$)$ & $<0.0001$ & $0.52(0.43$ to 0.62$)$ & $<0.001$ \\
\hline Non-events & $-0.17(-0.18$ to -0.15$)$ & $<0.0001$ & $-0.29(-0.33$ to -0.26$)$ & $<0.001$ \\
\hline Total NRI & $0.07(0.01$ to 0.13$)$ & 0.0363 & $0.23(0.13$ to 0.33$)$ & $<0.001$ \\
\hline
\end{tabular}

$\mathrm{EPI}_{\mathrm{cr}, \mathrm{cysc}}$ formula reclassified participants into a risk category (or eGFR category in this case) less appropriately for the outcome (death vs. no death). Results of category-free analyses based upon 1 change in eGFR were similar in direction to the category-based NRI for the comparison be-

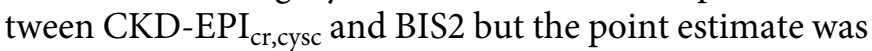
greater in magnitude and was statistically significant (table 4). Results of similar NRI comparisons between CKD$\mathrm{EPI}_{\text {cysc }}$ vs. CKD-EPI $\mathrm{cr}_{\mathrm{cr}, \mathrm{cysc}}$ found that CKD-EPI cysc $_{\text {led to }}$ more appropriate reclassification of eGFR for risk of death than inappropriate classification, though small in magnitude. In contrast, the CKD-EPI ${ }_{\mathrm{cr}}$ equation led to a greater proportion with inappropriate reclassification for their risk of death than appropriate reclassification when com-

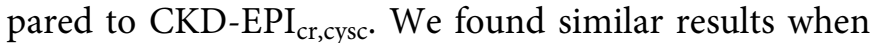
cardiovascular mortality was the outcome (table 4).

\section{Discussion}

We found that among community-dwelling older men, the BIS2 eGFR formula identified a higher proportion of participants as having CKD when compared to the CKD-EPI $I_{c r, c y s c}, C K D_{-} \mathrm{EPI}_{\text {cysc }}$, and CKD-EPI $\mathrm{cr}_{\mathrm{cr}}$ eGFR equations. This was largely due to identification of a higher proportion of participants with eGFR 45-59. In general, lower eGFR was associated with higher mortality, but the CKD-EPI equations were more sensitive and robust predictors of death than the BIS2 and CKD-EPI ${ }_{\mathrm{cr}}$ equations. Finally, when compared to $\mathrm{CKD}-\mathrm{EPI}_{\mathrm{cr}, \mathrm{cysc}}$, both the BIS2 and CKD-EPI ${ }_{\text {cr }}$ equations led to more inappropriate classification of participants with respect to mortality risk. For BIS2, this was driven by recategorization of participants who did not die to a lower eGFR. 
Taken together, our findings suggest that the BIS2 equation did not outperform and the CKD-EPI ${ }_{\mathrm{cr}}$ equation was inferior to the cystatin-C-based CKD-EPI equations to predict death among these community-dwelling older men.

The higher prevalence of eGFR $<60$ by the BIS2 (42\%) is consistent with what is reported in the literature for BIS2 eGFR and mGFR in community-dwelling older adults $[7,15,16]$. However, the BIS2 equation did not perform as well to predict death when compared to the SCysc-based CKD-EPI equations in this cohort of healthy older men. The first 3 explanations we present rest upon the assumption that the mortality risk predicted by eGFR is a direct result of the impact of renal function mGFR. First, in internal validation against iohexol mGFR, BIS2 performed better than the SCysc-based CKD-EPI and CKD-EPI ${ }_{\text {cr }}$ with respect to bias, precision and accuracy [7]. However, recent studies have shown variable performance upon external validation $[15,17,18]$. For example, in a study of 805 community-dwelling, older Icelandic men and women with mean iohexol GFR $62.4 \pm 16.5$, both the BIS2 and the SCr-based BIS equations had greater bias but similar precision and accuracy when compared to SCysc and SCr-based CKD-EPI equations [15]. In fact, both BIS equations had negative bias with respect to mGFR across all levels of renal function, but this was greater at higher mGFR [15]. This finding was echoed in another study of 394 Caucasian men and women with median iohexol mGFR 53 (IQR 7.2-100.9) and another of 95 Brazilian men and women with mean iohexol mGFR $55 \pm 15[17,18]$. The negative bias observed in these studies may in part explain the inappropriate reclassification to a lower eGFR by BIS2 compared to CKD-EPI cr,cysc $_{\text {with }}$ respect to risk of death. A second explanation for our findings is that, despite similarities in prevalence of CKD between MrOS Sleep and the BIS, other differences between our cohort and the BIS cohort affected the performance of BIS2 to predict death in MrOS Sleep. Indeed, the BIS cohort was only $58 \%$ male, while our cohort is entirely male [7]. In addition, our cohort had less DM and HTN compared to the BIS cohort (for MrOS vs. BIS 13 vs. $27 \%$ for DM and 63 vs. $78 \%$ for HTN) [7]. However, both cohorts consisted of community-dwelling adults who were entirely (BIS) or largely (MrOS Sleep) Caucasian with a mean age in the range 76-78 [7]. In contrast, the cohorts used to develop the CKD-EPI equations were younger (mean age $47 \pm 15$ ), more racially diverse with more DM and a greater spectrum of renal function than both the BIS and MrOS cohorts [5, 7]. Nevertheless, the performance of the SCysc-based CKD-EPI equations to predict mortality was superior to BIS2 in this cohort of older community-dwelling men. This suggests that differences between MrOS and the BIS participants are less likely to explain our findings. Third, it may also be that our choice of eGFR cutpoints for the categories may have somehow biased our findings against the BIS2 equation. However, re-examination of our data using eGFR in quartiles and category-free NRI yielded similar results suggesting that the selection of clinically relevant cutpoints for eGFR categories played a minimal role in our findings. Finally, the ability of each eGFR equation to predict death may be related to the prognostic value of the surrogate markers beyond that of mGFR. SCr is well known to reflect muscle mass [15]. In older adults, muscle mass reflects better health status and lower risk, but the resultant higher SCr results in lower eGFR and inappropriate allocation to a higher risk category. While SCysc may improve upon the limitations of SCr, it too may represent putative non-GFR determinants such as inflammation and fat mass or others that are as yet unknown [19]. Regardless, our primary focus was to compare the BIS2 equation, which incorporates both SCysc and SCr to CKD-EPI $I_{c r, c y s c}$. But for the absence of a race consideration by BIS2 that would be of little impact in the MrOS cohort of $90 \%$ Caucasian participants, both equations contain similar markers and considerations: SCysc and $\mathrm{SCr}$, age and gender. Thus, differences observed in performance were less likely related to non-GFR determinants of surrogate markers used but rather related to characteristics of the formula used.

Our findings have important clinical and research implications. The Kidney Disease Improving Global Outcomes (KDIGO) guidelines 2012 recommends use of SCysc-based eGFR when the eGFR is $45-59$ and there is no other evidence of renal damage such as albuminuria [20]. They further recommend the use of the CKDEPI equations or other equations that incorporate both SCr and SCysc if they are more accurate for a given population [20]. Community-dwelling older adults constitute a considerable proportion of $\mathrm{eGFR}_{\mathrm{cr}} 45-59$ without evidence of renal damage and thus would benefit from SCysc measurement for confirmation of CKD [20]. Furthermore, the 2014 Kidney Disease Outcomes Quality Initiative commentary on KDIGO suggested that SCyscbased measures improve upon SCr-based measures with respect to risk stratification [21]. It was therefore of great interest when the BIS provided the first SCyscbased equations developed specifically for communitydwelling older adults providing a potentially more appropriate equation for risk stratification in this popula- 
tion [7]. However, our data do not support the use of BIS2 over the SCysc-based CKD-EPI equations to predict mortality risk based upon renal function in older adults.

Our study is the first to report the performance of the BIS2 equation to predict mortality and to compare that performance to key existing eGFR formulae in a large cohort of community-dwelling older men. Other strengths of this study include the large sample size, availability of properly calibrated SCr and SCysc for estimation of renal function, detailed collection of covariate data, and extended follow-up time with minimal loss to follow-up. We also present a well-rounded assessment of discriminant ability of each equation to predict mortality, utilizing Cox-proportional hazards regression and NRI.

Despite these strengths, the MrOS study has some limitations. First, we cannot generalize the findings of this study to women, more diverse populations and individuals with moderate to severe CKD. However, with respect to $\mathrm{CKD}$, the BIS2 was developed in a cohort with very little CKD so that application of BIS2 to a communitydwelling cohort such as MrOS Sleep is appropriate [7]. Also, we did not have albuminuria available for this analysis. However, because our focus was to compare the performance of eGFR formulae against each other to predict death, albuminuria was less vital for this analysis. Finally, our analysis utilized an SCysc-based formula as the comparator for our analyses. There are very practical barriers to use of SCysc in the community related to the cost of testing, the need for calibration to high-level reference material and the sheer activation energy of changing laboratory reporting practices [21]. However, the price of testing is decreasing and the methodology for SCysc measurement is facilitated by the use of routinely available equipment [21]. As such, the era of routine SCysc reporting may be near. We believe it was important to use the CKD-EPI $\mathrm{cr}_{\mathrm{cr}, \mathrm{cysc}}$ as our key comparator based on recommendations by KDIGO to use CKD-EPI equations to estimate GFR, data suggesting SCysc-based equations better predict mortality in older adults, and because the

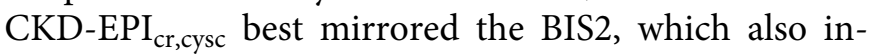
cludes SCr and SCysc [20,21].

In conclusion, our findings suggest that the BIS2, a new SCr/SCysc-based eGFR equation developed in healthy older adults, does not outperform the SCyscbased CKD-EPI equations to predict death in community-dwelling older men. In addition, the CKD-EPI $I_{c r}$ equation was inferior to the SCysc-based CKD-EPI equations to predict death in this cohort. For the purposes of mortality risk prediction in this population, the SCysc-based
CKD-EPI equations should be used. Future analyses should evaluate the ability of the BIS2 to predict death in other populations with a wide spectrum of renal function.

\section{Funding Source}

The Osteoporotic Fractures in Men (MrOS) Study is supported by National Institutes of Health funding. The following institutes provide support: the National Institute on Aging, the National Institute of Arthritis and Musculoskeletal and Skin Diseases, the National Center for Advancing Translational Sciences, and NIH Roadmap for Medical Research under the following grant numbers: U01 AG027810, U01 AG042124, U01 AG042139, U01 AG042140, U01 AG042143, U01 AG042145, U01 AG042168, U01 AR066160, and UL1 TR000128. The National Heart, Lung, and Blood Institute provides funding for the MrOS Sleep ancillary study 'Outcomes of Sleep Disorders in Older Men' under the following grant numbers: R01 HL071194, R01 HL070848, R01 HL070847, R01 HL070842, R01 HL070841, R01 HL070837, R01 HL070838, and R01 HL070839. Dr. M.T. Canales's time is supported by a VA CSR\&D Career Development Award (CX00053301A1) and by support from the Division of Nephrology, Hypertension and Renal Transplantation, Department of Medicine, University of Florida.

\section{Sponsor's Role}

None of the funding sources cited played any role in the design and conduct of the study; collection, management, analysis, and interpretation of the data; preparation, review, or approval of the manuscript; or the decision to submit the manuscript for publication.

\section{Acknowledgments}

This manuscript is also the result of work supported in part with resources and use of facilities at the Minneapolis VA Health Care System and the resources and facilities of the NF/SGVHS Medical Service, the Center of Innovation on Disability and Rehabilitation Research and the Geriatric Research Education Clinical Center, Gainesville, Fla., USA. The contents of this study do not represent the views of the US Department of Veterans Affairs or the United States Government.

The authors would also like to acknowledge Kyle Moen for his assistance in copyediting and formatting this manuscript and the corresponding author has received written consent to acknowledge Mr. K. Moen in this manuscript.

\section{Disclosure Statement}

The authors have nothing to disclose. 


\section{References}

1 Coresh J, Selvin E, Stevens LA, Manzi J, Kusek JW, Eggers P, Van Lente F, Levey AS: Prevalence of chronic kidney disease in the United States. JAMA 2007;298:2038-2047.

2 Foley RN, Murray AM, Li S, Herzog CA, McBean AM, Eggers PW, Collins AJ: Chronic kidney disease and the risk for cardiovascular disease, renal replacement, and death in the United States Medicare population, 1998 to 1999. J Am Soc Nephrol 2005;16:489-495.

3 Keith DS, Nichols GA, Gullion CM, Brown JB, Smith DH: Longitudinal follow-up and outcomes among a population with chronic kidney disease in a large managed care organization. Arch Intern Med 2004;164:659-663.

4 O'Hare AM, Choi AI, Bertenthal D, Bacchetti P, Garg AX, Kaufman JS, Walter LC, Mehta KM, Steinman MA, Allon M, McClellan WM, Landefeld CS: Age affects outcomes in chronic kidney disease. J Am Soc Nephrol 2007;18: 2758-2765.

5 Inker LA, Schmid CH, Tighiouart H, Eckfeldt JH, Feldman HI, Greene T, Kusek JW, Manzi J, Van Lente F, Zhang YL, Coresh J, Levey AS; CKD-EPI Investigators: Estimating glomerular filtration rate from serum creatinine and cystatin C. N Engl J Med 2012;367:20-29.

6 Shlipak MG, Matsushita K, Ärnlöv J, Inker LA, Katz R, Polkinghorne KR, Rothenbacher D, Sarnak MJ, Astor BC, Coresh J, Levey AS, Gansevoort RT; CKD Prognosis Consortium: Cystatin $\mathrm{C}$ versus creatinine in determining risk based on kidney function. $\mathrm{N}$ Engl J Med 2013;369:932-943.

7 Schaeffner ES, Ebert N, Delanaye P, Frei U, Gaedeke J, Jakob O, Kuhlmann MK, Schuchardt M, Tölle M, Ziebig R, van der Giet M, Martus P: Two novel equations to estimate kidney function in persons aged 70 years or older. Ann Intern Med 2012;157:471-481.
8 Inker LA, Eckfeldt J, Levey AS, LeiendeckerFoster C, Rynders G, Manzi J, Waheed S, Coresh J: Expressing the CKD-EPI (chronic kidney disease epidemiology collaboration) cystatin C equations for estimating GFR with standardized serum cystatin $\mathrm{C}$ values. Am J Kidney Dis 2011;58:682-684.

9 Blank JB, Cawthon PM, Carrion-Petersen ML, Harper L, Johnson JP, Mitson E, Delay RR: Overview of recruitment for the osteoporotic fractures in men study (MrOS). Contemp Clin Trials 2005;26:557-568.

10 Orwoll E, Blank JB, Barrett-Connor E, Cauley J, Cummings S, Ensrud K, Lewis C, Cawthon PM, Marcus R, Marshall LM, McGowan J, Phipps K, Sherman S, Stefanick ML, Stone K: Design and baseline characteristics of the osteoporotic fractures in men (MrOS) study - a large observational study of the determinants of fracture in older men. Contemp Clin Trials 2005;26:569-585.

11 Levey AS, Coresh J, Greene T, Stevens LA, Zhang YL, Hendriksen S, Kusek JW, Van Lente F; Chronic Kidney Disease Epidemiology Collaboration: Using standardized serum creatinine values in the modification of diet in renal disease study equation for estimating glomerular filtration rate. Ann Intern Med 2006; 145:247-254.

12 Ware J, Kosinski M, Keller S: SF-12: How to Score the SF-12 Physical and Mental Health Summary Scores. Linsoln, Quality Metric Inc., 1998.

13 Pencina MJ, D'Agostino RB Sr, D'Agostino RB Jr, Vasan RS: Evaluating the added predictive ability of a new marker: from area under the ROC curve to reclassification and beyond. Stat Med 2008;27:157-172; discussion 207-212.

14 Pencina MJ, D’Agostino RB Sr, Steyerberg EW: Extensions of net reclassification improvement calculations to measure usefulness of new biomarkers. Stat Med 2011;30:11-21.
15 Fan L, Levey AS, Gudnason V, Eiriksdottir $\mathrm{G}$, Andresdottir MB, Gudmundsdottir $\mathrm{H}$, Indridason OS, Palsson R, Mitchell G, Inker LA: Comparing GFR estimating equations using cystatin $C$ and creatinine in elderly individuals. J Am Soc Nephrol 2015;26:19821989.

16 Montesanto A, De Rango F, Berardelli M, Mari V, Lattanzio F, Passarino G, Corsonello A: Glomerular filtration rate in the elderly and in the oldest old: correlation with frailty and mortality. Age (Dordr) 2014;36:9641.

17 Alshaer IM, Kilbride HS, Stevens PE, Eaglestone G, Knight S, Carter JL, Delaney MP, Farmer CK, Irving J, O'Riordan SE, Dalton RN, Lamb EJ: External validation of the Berlin equations for estimation of GFR in the elderly. Am J Kidney Dis 2014;63:862-865.

18 Lopes MB, Araújo LQ, Passos MT, Nishida SK, Kirsztajn GM, Cendoroglo MS, Sesso RC: Estimation of glomerular filtration rate from serum creatinine and cystatin $\mathrm{C}$ in octogenarians and nonagenarians. BMC Nephrol 2013; $14: 265$

19 Knight EL, Verhave JC, Spiegelman D, Hillege HL, de Zeeuw D, Curhan GC, de Jong PE: Factors influencing serum cystatin $\mathrm{C}$ levels other than renal function and the impact on renal function measurement. Kidney Int 2004;65:1416-1421.

20 KDIGO 2012 clinical practice guideline for the evaluation and management of chronic kidney disease: Kidney Int Suppl (2011) 2013; 3:1-150.

21 Inker LA, Astor BC, Fox CH, Isakova T, Lash JP, Peralta CA, Kurella Tamura M, Feldman HI: KDOQI US commentary on the 2012 KDIGO clinical practice guideline for the evaluation and management of CKD. Am J Kidney Dis 2014;63:713-735. 